\title{
Development of a Laser Phase Plate for Zernike Phase Contrast in Electron Microscopy
}

\author{
Michelle Xu ${ }^{1}$, Erin Sohr ${ }^{1}$, Brian Shevitski ${ }^{1}$, Robert M. Glaeser ${ }^{2}$, and Holger Müller ${ }^{1,3}$ \\ 1. Department of Physics, University of California, Berkeley, CA 94720 USA \\ 2. Life Sciences Division, Lawrence Berkeley National Laboratory, University of California, Berkeley, \\ CA 94720 USA \\ 3. Chemical Sciences Division, Lawrence Berkeley National Laboratory, University of California, \\ Berkeley, CA 94720 USA
}

Phase contrast for transmission electron microscopy (TEM) was originally proposed by Boersch in 1947 [1]. Unlike in light microscopy, it has been difficult to develop a phase-shifting $\lambda / 4$-plate for an electron beam that does not attenuate or distort the image, and that exhibits a long lifetime. Approaches that have been tried include thin carbon films, electrostatic phase plate based on thin wires and microfabricated electrodes, and zone plates [2].

We present our project to build a photonic phase plate, in which the interaction of the electron beam with an intense laser focus will be used to phase shift the electron wave function [3]. The laser phase plate does not require the electron beam to pass through, or even come close to, any physical barrier, and should thus be free from problems with lifetime and electrostatic charging of surface contamination. However, the required laser power of several kilowatts will be hard to achieve. Resonant cavities are required, but available state of the art cavities do not provide the required focusing of the laser radiation with a numerical aperture of NA $>1 / 2$.

Theory based on quantum electrodynamics, in analogy to the Compton effect, predicts the phase shift as $\delta=\frac{\pi}{2} \frac{1}{\beta \gamma}\left(\frac{P}{\mathrm{~kW}}\right)\left(\frac{\lambda}{\mu \mathrm{m}}\right) N$,

where $\beta=v / c$ and $\gamma=1 /\left(1-v^{2} / c^{2}\right)^{1 / 2}$ are the Lorentz factors for an electron velocity $v, P$ is the laser power, $\lambda$ the laser wavelength, and $N$ a numerical factor that depends on the geometry of the focusing optics [3].

We have studied two approaches: The first is a plano-parabolic optical cavity, formed by a near-plane low-loss dielectric mirror and a gold-coated parabolic mirror (Fig. 1). Strong focusing with an NA approaching $\pi$ and resonant power enhancement reduces the required laser power and allows working with a long wavelength $(\lambda=10.6 \mu \mathrm{m})$, which reduces the needed power further while still reaching the required spot size of $\sim 2 \mu \mathrm{m}$. The factor $N=0.15 /(1-r)$, where $r=90-98 \%$ is the effective intensity reflectivity of the parabolic mirror (taking into account losses at the aperture of the mirror). Simulations (Fig. 1) show that a radially polarized incident beam leads to best focusing. A commercial $\mathrm{CO}_{2}$ laser has been modified to obtain the required radially polarized, ring-shaped mode. To verify the predicted intensity and size of the focus, we will use the focus as a far-detuned optical dipole trap for cesium atoms. Measurement of the ac Stark effect and the resonance frequency of the atoms in the trap will reveal the intensity and size of the focus. The plano-parabolic cavity, while being viable, will still require large laser power $(\sim 100 \mathrm{~W})$ and result in a focus large enough $(\sim 0.3 \lambda$ diameter $)$ to reduce image contrast for low spatial frequencies.

The second approach is based on a Fabry-Perot cavity with highly reflective dielectric mirrors. The 
factor $N=0.0075 \times \mathrm{NA} /(1-r)$ can be large thanks to a large of $r \sim 99.99 \%$. This will allow operation at $\lambda=1.064 \mu \mathrm{m}$ and, hence, smaller foci while reducing the required laser power. However, a numerical aperture $\mathrm{NA} \equiv r_{\mathrm{m}} / R$, where $r_{\mathrm{m}}$ is the radius of the mirror and $R$ its radius of curvature, of $\sim 0.5$ is required. We are working with a coating company (Layertec) to develop suitable mirrors. The cavity will be nearly concentric, with a mirror spacing of $L=2(R-\epsilon)$, where $\epsilon \ll R$. To obtain a near-Gaussian TEM00 mode having the needed NA, an $\epsilon$ of a few nanometers will have to be maintained. This will be achieved by a dual feedback mechanism: it stabilizes the resonance frequency of the cavity to the frequency of the laser which, in turn, is adjusted such that a constant NA is maintained. This feedback will also compensate for changes in the mirror radius of curvature that result from heating of the mirrors by the laser power [4].

Construction of the optomechanical setup to join the laser optical phase plate and an existing FEI Titan 80-300 keV electron microscope has begun (Fig. 2).

\section{References:}

[1] H Boersch, Z. Naturforschg. 2a (1947), p. 615

[2] K Nagayama, J. Electron Microscopy 60 (2011), p. S43.

[3] H Müller et al., New J. Phys. 12 (2010), p. 073011.

[4] L S Meng, J K Brasseur, and D K Neumann, Optics Express 13 (2005), p. 10085

The authors acknowledge funding from the U. S. National Science Foundation. We thank J. Jin, R. Danev, J. Spence, and H. Padmore for many useful discussions and contributions to this work.
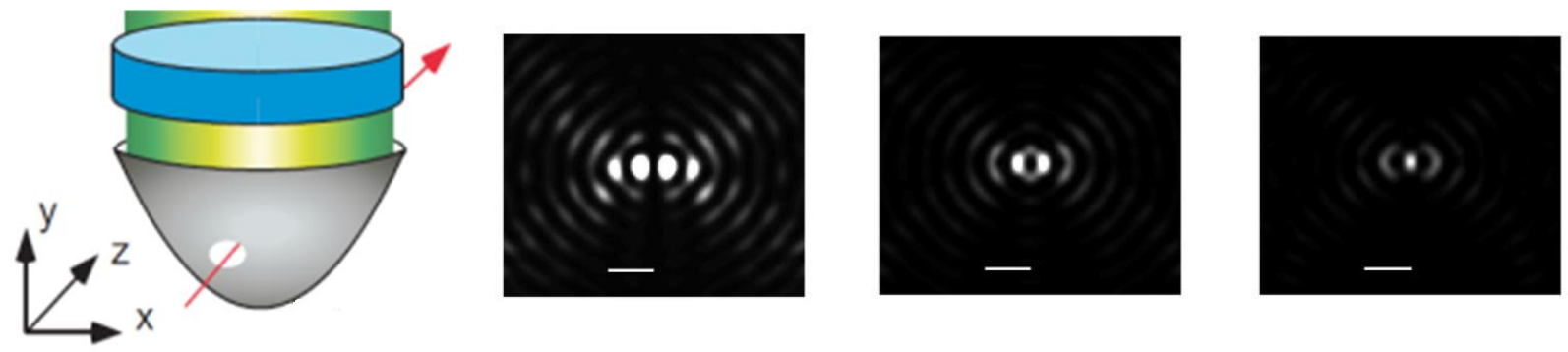

Figure 1. Left: Plano-parabolic cavity. The electron beam (red) passes the focus. Right: simulated intensities $|E|^{2}$ in the focal plane for different incident polarizations. From left to right: azimuthal, linear, and radial. The scale bar denotes one optical wavelength. Radial polarization results in the smallest focal point and is thus preferred.

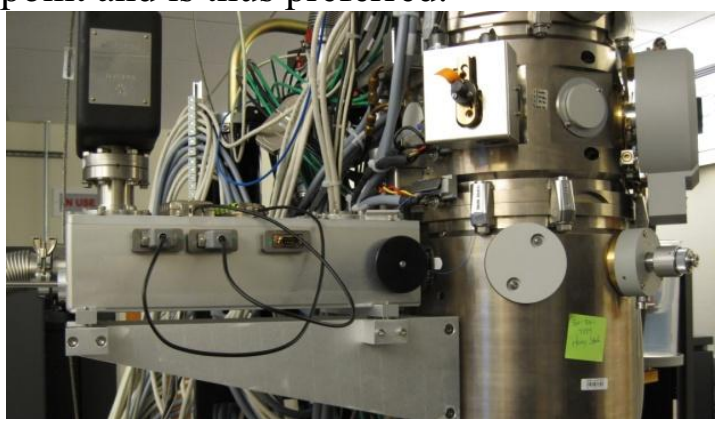

Figure 2. FEI Titan 8-300 keV electron microscope with a phase plate holder (under vacuum) of a previous project. A similar setup will hold the optical components and opto-mechanical actuators to position the focus of the laser relative to the electron beam and stabilize the resonance frequency of the cavity. 